\title{
Analysis of chiral amino acids in conventional and transgenic maize
}

Miguel Herrero, Elena Ibáñez, Pedro J. Martín-Álvarez, Alejandro Cifuentes*

Institute of Industrial Fermentations (CSIC), Juan de la Cierva 3, 28006 Madrid, Spain.

*Corresponding author: Dr. Alejandro Cifuentes, Fax\#: 34-91-5644853, e-mail: acifuentes@ifi.csic.es 


\section{ABSTRACT}

In this work, a new chiral micellar electrokinetic chromatography with laser induced fluorescence detection (chiral-MEKC-LIF) method is proposed to identify and quantify Dand L-amino acids in three lines of transgenic maize and their corresponding nontransgenic parental lines grown under identical conditions. The optimized procedure includes amino acids extraction, derivatization with FITC and chiral-MEKC-LIF separation in a background electrolyte composed of $100 \mathrm{mM}$ sodium tetraborate, $80 \mathrm{mM}$ SDS, $20 \mathrm{mM} \beta-\mathrm{CD}$ at $\mathrm{pH}$ 10.0. The D- and L-forms of Arg, Ser, Ala, Glu and Asp, corresponding to the majority amino acids usually found in maize, are separated in less than 25 min with efficiencies up to 890,000 plates/m and high sensitivity (i.e., LODs as low as $160 \mathrm{nM}$ were obtained for D-Arg for a signal to noise ratio of three), allowing the detection of $1 \%$ of D-Arg in the presence of $99 \%$ of its optical antipode. Using this method, different D-amino acids are detected in all investigated maize samples providing the reproducible quantification of the D-enantiomeric excess (\%D-aa) for each amino acid calculated as $\% \mathrm{D}-\mathrm{aa}=100 \cdot \mathrm{D}-\mathrm{aa} /(\mathrm{D}-\mathrm{aa}+\mathrm{L}-\mathrm{aa})$. Thus, significant differences were observed among the \%D-aa values for the different conventional varieties (Aristis, Tietar and PR33P66 maize) as could be expected from their natural variability. More interestingly, comparing each conventional maize with its corresponding transgenic line, very similar $\% \mathrm{D}$-aa values were obtained for one of the studied maize couples (Tietar vs. Tietar-Bt) what could be presented as a new proof of their substantial equivalence. However, significant differences in the \%D-aa values were observed for the other lines of maize studied. It is concluded that enantioselective procedures can open new perspectives in the study of transgenic organisms in order to corroborate (or not) the equivalence with their conventional counterparts. To our knowledge, this is the first chiral report investigating the chemical composition of genetically modified crops. 